\title{
Linking STAT and TLR signaling in microglia: a new role for the histone demethylase Jmjd3
}

\author{
Uwe-Karsten Hanisch
}

Published online: 7 February 2014

(C) Springer-Verlag Berlin Heidelberg 2014

Inflammation is an essential process in the immune defense against infections. It involves concerted, timed, and highly regulated activities of tissue-resident and infiltrating immune cells, their soluble messengers, and effector molecules to fight off an invasion as well as to support subsequent attempts of repair. Inflammatory processes can also develop under noninfectious conditions triggered by some endogenous factors and as a consequence of cell and tissue impairment. Here too, the initiation of activities, namely in innate immune cells, would primarily aim at damage containment, tissue protection, and recovery. However, excessive, chronic or maladapted inflammation can precipitate destructive cascades, which can eventually result in organ failure or even lead to a lethal outcome. In this regard, the central nervous system (CNS) is extremely vulnerable.

Neuroinflammatory processes as they follow and accompany not only viral and bacterial infections, trauma, or stroke, but also neurodegenerative and autoimmune diseases can have a devastating impact on neuronal as well as glial structures and impede their sophisticated functions often causing massive and lasting disabilities $[1,2]$. Therefore, it has been of foremost interest to establish therapeutic strategies which would allow for moderating dysregulated inflammatory reactions, especially also those affecting the CNS [2-5]. However, some of the interventions can cause themselves undesired effects - increasing the need to develop improved anti-inflammatory compounds and, in addition, to identify novel targets within the (pro)inflammatory signaling and effector mechanisms.

Now and again, studies unravel links and interactions between signaling systems which, at first glance, do not intimately cooperate. Some reveal new functions of proteins

U.-K. Hanisch $(\bowtie)$

Institute of Neuropathology, University of Göttingen,

Robert-Koch-Straße 40, 37075 Göttingen, Germany

e-mail: ukhanisch@med.uni-goettingen.de that reach beyond of what was making its way to the textbooks. The work of Przanowski and collaborators [6] combines both kinds of surprises by linking Janus kinase-signal transducers and activators of transcription (JAK-STAT) signaling elements closely to the world of Toll-like receptors (TLRs). Not enough, an enzyme known for removing methyl groups from lysine 27 residues in $\mathrm{H} 3$ histones, the H3K27me3 demethylase Jmjd3, appears to be a key element in this cooperation - yet not by its enzymatic activity but as a transcription factor. The findings bear (neuro)inflammation-relevant novelty as they are at the core of innate immune functions in the CNS.

Within the CNS, microglial cells represent the equivalent of a tissue macrophage [7]. While having contributions to the CNS development and functional maintenance, these myeloid cells also serve as sentinels of the tissue homeostasis constantly scanning their environment and being capable of instantly responding to signs of disturbance with the functional repertoire of an innate immune cell [8]. Their versatile response options are currently gaining enormous attention also for clinical implications. There is virtually no neuropathological process which would take place without microglial reactions or active involvement.

As innate immune cells, microglia are equipped with an array of receptors that enable them to sense any threat to the structural and functional integrity of the CNS [7]. Members of the TLR family thereby simultaneously serve the recognition of infection and damage [9]. As pattern recognition receptors (PRRs), they sense an assortment of evolutionary conserved structural motifs in the RNA, DNA, glycoproteins, and glycolipids of microbes, termed pathogen-associated molecular patterns (PAMPs). Intriguingly, the very same receptors manage to signal upon binding of self-derived molecules, mainly proteins, but also lipids, which usually serve most diverse functions within cells, the extracellular matrix or the circulation. Disparate by biochemical structure and implementation, they 
are alike by gaining the meaning of a damage- or dangerassociated molecular pattern (DAMP) upon translocation to non-physiological compartments and/or molecular modification [10]. Even though TLRs are by far not the only molecular antennas serving microglial surveillance duties, they can claim to cover both the identification of essential pathogens as well as the detection of representative signs of deterioration - and to induce the production of a plethora of cytokines and chemokines as messengers to orchestrate appropriate responses for defense and restoration. Yet some endogenous TLRagonistic factors may also drive rather detrimental responses and fuel destructive events $[11,12]$.

Przanowski and coworkers identified previously unknown STAT target genes in TLR4 agonist-stimulated microglia [6]. TLR4 is best known for the binding and signaling of lipopolysaccharide (LPS), a major cell wall component of gramnegative bacteria and a prototypical PAMP. On the other hand, TLR4 also recognizes a variety of DAMPs. So, some of the findings made for LPS could have similar importance for endogenous TLR agonists, even though PAMP and DAMP signaling consequences can differ substantially.

Among the novel STAT1- and STAT3-targeted genes, the authors found the JmjC-domain protein Jmjd3. Jmjd3 had already been associated with inflammatory responses, i.e., in macrophage activation by LPS [13]. Its own expression is induced by (and actually dependent on) NFKB, a key transcription factor downstream of TLRs. Przanowski and collaborators now provide convincing (and multiple) evidence that Jmjd3 can also be induced by STAT1 and STAT3, preferentially by their combined activation. Moreover, Jmjd3 now reveals transcription factor activity on its own and synergizes with the STATs in driving a number of proinflammatory cytokines and chemokines, including TNF $\alpha$, IL-1 $\beta$, IL-6, IFN $\gamma$, and CCL5. Notably, demethylase activity of Jmdj3 is dispensable for this function. Experiments combining overexpression of constitutively active STATs with Jmjd3 resulted in amounts of cytokine production matching the levels obtained with LPS. Although the actual profiles of STAT1/3- and LPSinducible genes were not congruent, they showed substantial overlap. Furthermore, silencing, supernatant transfer, and protein synthesis block approaches indicate that STAT activation is part of the LPS $\rightarrow$ TLR4-triggered cascade to the induction of (several) cytokines - and that the TLR4-driven release of (some) factors actually creates a loop which is responsible for STAT1 and STAT3 phosphorylation. In a summarizing hypothesis, the various elements are integrated to draw a scenario in which TLR4 activation in microglia causes an NFKBdependent induction of cytokines which, upon release, organize for STAT1 and STAT3 phosphorylation and transcriptional activity. The NFKB and the STAT pathways would both upregulate Jmjd3, which in turn would further cooperate with them to govern the production of the full spectrum of proinflammatory mediators (Fig. 1).
The comprehensive model is tempting. Microglia are endowed with a battery of TLRs and cytokine receptors. While the JAK-STAT system is not considered as a prototypical pathway in TLR signaling, both domains are now brought together - and a demethylase activity-independent contribution of Jmjd3 is placed in this network. Jmjd3 would serve therein as a target as well as act as an active player.

The work of Przanowski and colleagues also raises several (new) questions. Which of the numerous TLR4-induced cytokines are responsible for the STAT-mediated transcription of Jmjd3 and inflammatory genes? Candidates should fulfill a few key criteria. Such cytokines should be themselves induced early enough upon TLR stimulation, which applies to many if not most of them, even though individual time courses of effective release can differ. Is this time frame of release sufficient to explain the induction of other gene products simply by an autocrine loop? In other words, is the TLR-triggered cytokine production and subsequent signaling via their own receptors and through STAT activation rapid enough to organize for the response? Przanowski et al. identify phosphorylated forms of STAT1, STAT3, and STAT5 in microglia already $1 \mathrm{~h}$ after the onset of LPS exposure. This is very fast, considering transcription, translation, and release of a factor that should bind to a JAK-driving receptor beforehand. Is the observed STAT activation thus a truly secondary phenomenon? Which of the TLR-induced factors are then controlled directly, which depend on indirect inductions? Is the TLR response required in parallel, or is it in some cases reduced to an initiation event?

The critical cytokines carrying the autocrine loop should drive STAT1 and STAT3 activation. IL-6 is thus a likely candidate. Signaling through the gp130 unit of its receptor leads to a phosphorylation of STAT3 [14]. IL-6 can also activate STAT1 and STAT5, matching the pattern observed by Przanowski et al. in their microglia challenge with LPS. Yet IL-6 is not the only member of its family, and STAT1 activation could also be assigned to interferons, such as IFN $\beta$ (which is induced under TLR4 though the TRIF signaling pathway).

If certain (critical) cytokines mediate the induction of other inflammatory genes upon microglial TLR activation, such cytokines could also be derived from other cells. Astrocytes present with a more limited spectrum of TLR-driven cyto- and chemokines (as they may not respond to all TLR agonists or release only a meager variety of factors compared to microglia). Nevertheless, appropriate stimuli could still cause some astrocytic contributions. Could astrocytes thereby influence microglial release phenotypes? If not astrocytes, other cellular sources can be imagined. In a situation of TLR activation, i.e., either an infectious challenge or a condition of 'sterile' tissue damage, immune cell infiltrates would enrich the environment with their own release products including cytokines (and interferons) that are suggested by the study of Przanowski et al. to engage with the execution of a full microglial response to TLR. 


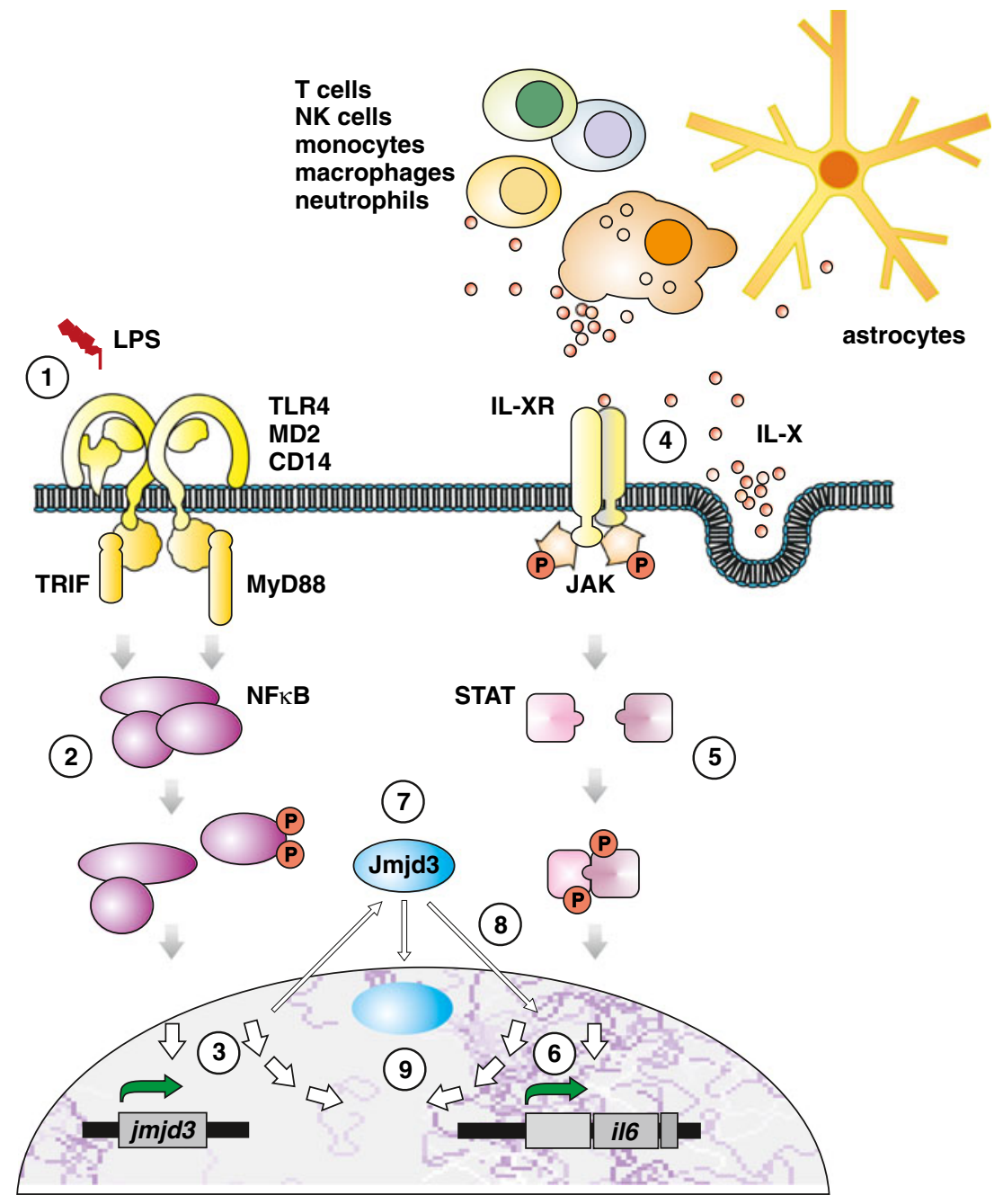

Fig. $1 \mathrm{Jmjd} 3$ as an integral signaling element in microglial responses to TLR activation. A challenge of microglial cells by LPS causes activation of TLR4 and signaling through pathways depending on the two adaptor proteins TRIF and MyD88 (I). Downstream cascades involve several kinases which activate transcription factors, such as NFKB (2), to finally induce gene transcription (3). TLR4 stimulation thereby results in the production and release of a plethora of cytokines and chemokines with important immunoregulatory activities, including TNF $\alpha$, IL-6, or IFN members (4). These factors can recruit and influence immune as well as resident cells, respectively. Certain factors may also participate in auto- or paracrine loops to affect the performance of microglia. Cytokines, like IL-6, or IFN $\beta$ could thus activate receptors linked to JAK family members, which in turn activate (i.e., phosphorylate) STAT proteins (5). Upon homo- and heterodimerization, STATs would also drive gene transcription (6). Based on the findings of Przanowski et al. [6], Jmjd3 (7) would be induced via NFkB-dependent as well as STAT-dependent pathways $(3,6)$ while serving itself as a transcription factor for the induction of proinflammatory cyto- and chemokines (8). Interestingly, this transcription factor role does not require the histone demethylase activity, which has been assigned

One may also raise the question whether this mechanism applies to other TLRs as well. TLR4 is in the privileged situation to rely on both TLR signaling pathways as they depend on the adaptor proteins TRIF and MyD88. Most TLR members have only access to the MyD88 route, whereas TLR3 exclusively uses TRIF. Restricted involvement of one to Jmjd3 as its primary function thus far. Yet once induced, histone modifications by Jmjd3 may still be part of its functional portfolio and play a role in lasting adjustments upon TLR4 activations (9). While a link between TLR $\rightarrow \mathrm{NFKB}$ and cytokine receptor/JAK $\rightarrow$ STAT signaling for the synthesis of a cyto- and chemokine profile could be nicely explained by the induction of Jmjd3 as an essential transcription factor, the model still leaves a few questions unanswered. One would regard the time scale for the activation of STATs. Effective release of cytokines upon TLR4 activation ( 1 to 4 ) and subsequent autocrine signaling through their receptors for STAT-dependent Jmjd3 induction (5 to 7) would take time before Jmjd3 could join as a transcription factor for further cytokine production (8). On the other hand, cytokines (including also IFN $\gamma$ ) being potentially relevant for driving Jmjd3 induction (4) may also derive from other cellular sources, including infiltrating immune (e.g., T and NK cells, monocytes/ macrophages, or neutrophils) and CNS-resident cells (e.g., astrocytes). Finally, the model shown here for LPS as a prototypical PAMP may also apply to TLR4 activation by DAMP molecules, and other TLR could reveal a similar interaction with STAT-driven Jmjd3 functions. (Partially adapted from Przanowski et al. [6])

or the other pathway would dictate the set of induced cytokines which would provide the loop to the STAT activation.

Finally, although Jmjd3 inserts in such a model largely without its demethylase activity, its ability to participate in the control of TLR(4)-mediated immediate responses may not exclude important epigenetic functions as to histone 
modulation. After all, transcriptional regulation of and via Jmjd3 may also have more long-term consequences for microglia. Considering them as a long-lived population within the CNS, acute, transient, or repeated challenges of microglia through their TLR system - by PAMPs and DAMPs - could leave traces and affect their responsiveness and responses, especially in aging-associated disease processes. Taken together, TLRs and cytokine receptors, NFKB and STATs, are getting closer and intermingled, some of their downstream elements and targets even assigned upstream function for the induction of others. Understanding inflammatory signaling in more detail was and will be an important task in microglial research and beyond.

\section{References}

1. Perry VH, Cunningham C, Holmes C (2007) Systemic infections and inflammation affect chronic neurodegeneration. Nat Rev Immunol 7: 161-167

2. Murray PJ, Smale ST (2012) Restraint of inflammatory signaling by interdependent strata of negative regulatory pathways. Nat Immunol 13:916-924

3. McGeer PL, McGeer EG (2007) NSAIDs and Alzheimer disease: epidemiological, animal model and clinical studies. Neurobiol Aging 28:639-647

4. Ghoreschi K, Laurence A, O'Shea JJ (2009) Selectivity and therapeutic inhibition of kinases: to be or not to be? Nat Immunol 10:356360
5. Schweingruber N, Reichardt SD, Luhder F, Reichardt HM (2012) Mechanisms of glucocorticoids in the control of neuroinflammation. J Neuroendocrinol 24:174-182

6. Przanowski P, Dabrowski M, Ellert-Miklaszewska A, Kloss M, Mieczkowski J, Kaza B, Ronowicz A, Hu F, Piotrowski A, Kettenmann H et al (2013) The signal transducers Stat1 and Stat3 and their novel target Jmjd3 drive the expression of inflammatory genes in microglia. J Mol Med (Berl). doi:10.1007/s00109-0131090-5

7. Kettenmann H, Hanisch UK, Noda M, Verkhratsky A (2011) Physiology of microglia. Physiol Rev 91:461-553

8. Hanisch UK, Kettenmann H (2007) Microglia: active sensor and versatile effector cells in the normal and pathologic brain. Nat Neurosci 10:1387-1393

9. Kawai T, Akira S (2010) The role of pattern-recognition receptors in innate immunity: update on Toll-like receptors. Nat Immunol 11: 373-384

10. Zhang X, Mosser DM (2008) Macrophage activation by endogenous danger signals. J Pathol 214:161-178

11. Stewart CR, Stuart LM, Wilkinson K, van Gils JM, Deng J, Halle A, Rayner KJ, Boyer L, Zhong R, Frazier WA et al (2010) CD36 ligands promote sterile inflammation through assembly of a Toll-like receptor 4 and 6 heterodimer. Nat Immunol 11:155-161

12. Lehnardt S, Schott E, Trimbuch T, Laubisch D, Krueger C, Wulczyn G, Nitsch R, Weber JR (2008) A vicious cycle involving release of heat shock protein 60 from injured cells and activation of toll-like receptor 4 mediates neurodegeneration in the CNS. J Neurosci 28: 2320-2331

13. De Santa F, Totaro MG, Prosperini E, Notarbartolo S, Testa G, Natoli G (2007) The histone H3 lysine-27 demethylase Jmjd3 links inflammation to inhibition of polycomb-mediated gene silencing. Cell 130: 1083-1094

14. Aaronson DS, Horvath CM (2002) A road map for those who don't know JAK-STAT. Science 296:1653-1655 